\title{
Is there a neuroanatomical basis of the vulnerability to suicidal behavior? A coordinate-based meta-analysis of structural and functional MRI studies
}

\author{
Kees van Heeringen*, Stijn Bijttebier, Stefanie Desmyter, Myriam Vervaet and Chris Baeken
}

Unit for Suicide Research, Department of Psychiatry and Medical Psychology, Ghent University, Ghent, Belgium

\section{Edited by:}

Srikantan S. Nagarajan, University of California, San Francisco, USA

\section{Reviewed by:}

Michael Minzenberg, University of California Davis School of Medicine, USA

Qiyong Gong, West China Hospital of Sichuan University, China

\section{*Correspondence:}

Kees van Heeringen, Unit for Suicide Research, Department of Psychiatry and Medical Psychology, Ghent University, University Hospital 1K12F, De Pintelaan 185, Gent 9000,

Belgium

e-mail: cornelis.vanheeringen@ ugent.be

\begin{abstract}
Objective: We conducted meta-analyses of functional and structural neuroimaging studies comparing adolescent and adult individuals with a history of suicidal behavior and a psychiatric disorder to psychiatric controls in order to objectify changes in brain structure and function in association with a vulnerability to suicidal behavior.
\end{abstract}

Methods: Magnetic resonance imaging studies published up to July 2013 investigating structural or functional brain correlates of suicidal behavior were identified through computerized and manual literature searches. Activation foci from 12 studies encompassing 475 individuals, i.e., 213 suicide attempters and 262 psychiatric controls were subjected to meta-analytical study using anatomic or activation likelihood estimation (ALE).

Result: Activation likelihood estimation revealed structural deficits and functional changes in association with a history of suicidal behavior. Structural findings included reduced volumes of the rectal gyrus, superior temporal gyrus and caudate nucleus. Functional differences between study groups included an increased reactivity of the anterior and posterior cingulate cortices.

Discussion: A history of suicidal behavior appears to be associated with (probably interrelated) structural deficits and functional overactivation in brain areas, which contribute to a decision-making network. The findings suggest that a vulnerability to suicidal behavior can be defined in terms of a reduced motivational control over the intentional behavioral reaction to salient negative stimuli.

Keywords: suicide, vulnerability, meta-analysis, gray matter, decision-making

\section{INTRODUCTION}

It is estimated that one million people commit suicide annually (World Health Organisation [WHO], 2002). Non-fatal suicidal behavior occurs 10-20 times more frequently, and a history of self-harm or suicide attempts is the strongest risk factor for suicide, being present in at least $40 \%$ of cases (Hawton and van Heeringen, 2009). Human and societal costs of suicidal behavior are substantial, and many countries have recently developed suicide prevention programs. The prevention of suicide, however, still poses major challenges. Clinicians are unable to predict the occurrence of suicidal behavior. In addition, when suicide risk is considered high, its management proves challenging because of the poor evidence base. It is, for example, impossible to predict whether an individual at risk will respond to treatment with a decrease or an increase in suicide risk. Even if there is a positive response to treatment, it is often unclear how and why this happened. Suicide prevention is thus in great need of markers that predict suicidal behavior and serve as a substrate for treatment.

Based upon the current state of knowledge, a stress-diathesis or stress-vulnerability model of suicidal behavior has been developed (Van Heeringen, 2012). In general, stress-vulnerability models describe the mechanisms due to which diathetic or vulnerable individuals respond with abnormal or pathological reactions to physiological stimuli or the ordinary conditions of life that are borne by the majority of individuals without injury (Zuckerman, 1999). With regard to suicidal behavior, stressors including interpersonal, professional, or financial problems commonly precipitate these behaviors. In addition, suicidal behavior occurs, in general, in the context of psychiatric conditions such as depression or bipolar disorder. However, only a small proportion of individuals confronted with stressors and suffering from these disorders will actually show suicidal behavior. The stressvulnerability model of suicidal behavior may thus explain such behavior as the consequence of an interaction between exposure to stressors and a vulnerability in individuals suffering from psychiatric disorders. Comparing individuals with a history of a psychiatric disorder and suicidal behavior to those with a history of such a psychiatric disorder but no history of suicidal behavior can thus be expected to shed light on the particular vulnerability to suicidal behavior.

Evidence of a neurobiological basis of the vulnerability to suicidal behavior is increasing (van Heeringen and Mann, 2014). From a neurochemical point of view, the serotonin neurotransmission system and the HPA-stress-response system appear to be crucially involved. At a neuroanatomical level, postmortem and neuroimaging studies show changes in a number of areas in the 
brain in association with a vulnerability to suicidal behavior. Four reviews of neuroimaging studies have recently been published (Desmyter et al., 2011; Jollant et al., 2011; van Heeringen et al., 2011; Zhang et al., 2014). These reviews agreed on the involvement of particular brain areas in the development of suicidal behavior, including the dorsolateral and orbitofrontal cortex. However, no clear picture emerged from the reviews with regard to other cortical regions and subcortical involvement. Moreover, sample sizes of studied patient groups were small, which hampers the interpretation of findings.

In order to investigate whether the subjective conclusions from the reviews can be objectified, and, more importantly, to specifically study the vulnerability to suicidal behavior using neuroimaging, we performed coordinate-based meta-analyses of structural and functional neuroimaging studies (CBMA; Turkeltaub et al., 2002). The objective of CBMA is not to estimate the magnitude of an effect across studies, but rather to identify anatomical locations in which an effect is observed consistently. By including only studies in which brain structure or function of individuals with a history of a psychiatric disorder and suicidal behavior were compared to brain structure or function of those with a history of the psychiatric disorder but no suicidal behavior, this meta-analysis aimed at investigating particularly the association between a vulnerability to suicidal behavior and brain structure or function.

\section{MATERIALS AND METHODS DATA SEARCH}

A search was carried out using Web of Science, PsycINFO, and Pubmed databases with the keywords: suicide, suicidal, neuroimaging, magnetic resonance imaging (MRI), positron emission tomography (PET), single photon emission tomography (SPET), single photon emission computerized tomography (SPECT), voxel based morphometry (VBM). Criteria for including studies in the meta-analyses were:

(1) Peer-reviewed original structural or functional brain imaging study published up to July 2013.

(2) Use of PET, SPE(C)T or MRI.

(3) Contrast between individuals with a history of a psychiatric disorder and suicidal behavior and individuals with a history of the psychiatric disorder but no suicidal behavior

(4) Adolescents and adults.

(5) Results reported as coordinates in a normalized standard stereotactic space, i.e., the Talairach or Montreal Neurological Institute (MNI) reference system.

Unpublished studies, case reports or conference abstracts were not included, as were studies reporting only region-of-interest findings or using seed-voxel-based analysis procedures. Studies comparing elderly suicide attempters to elderly psychiatric controls were not included in the meta-analysis, because the neurobiology of suicidal behavior may differ between the elderly and adolescents or adults. The reference lists of relevant papers were checked manually for additional relevant publications not previously identified.

The literature search identified articles reporting on 22 structural and 16 functional brain imaging studies, in which individuals with a history of suicide attempts were included and compared to controls.

Seven structural imaging studies were excluded because Talairach or MNI coordinates of identified anatomical structures were not reported (Monkul et al., 2007; Matsuo et al., 2010; Vang etal., 2010; Cyprien etal., 2011; Spoletini etal., 2011; NeryFernandes etal., 2012; Giakoumatos et al., 2013). Two studies were excluded because the study population consisted of elderly (Hwang et al., 2010; Dombrovski et al., 2012). Two additional studies were excluded because results for suicide attempters were not reported separately as attempters were regarded as part of a larger group of individuals considered at increased risk of suicide (Wagner et al., 2011,2012). Finally, five studies were excluded from the meta-analysis because they focused specifically on hyperintensities, i.e., white matter hyperintensities (Ahearn et al., 2001) or gray matter hyperintensities (Ehrlich et al., 2004, 2005; Pompili et al., 2007, 2008).

Identified functional imaging studies used SPECT $(n=4$; Audenaert et al., 2001, 2002; Lindstrom et al., 2004; Ryding et al., 2006), PET ( $n=4$; Meyer et al., 2003; Oquendo et al., 2003; Leyton etal., 2006; Nye et al., 2013), and fMRI $(n=8$; Jollant et al., 2008, 2010; Pan et al., 2011, 2013a,b; Marchand et al., 2012; Dombrovski et al., 2013; Fan et al., 2013). A number of studies had to be excluded from the meta-analysis due to absence of psychiatric controls (Audenaert et al., 2001, 2002; Oquendo et al., 2003; Leyton et al., 2006), or the absence of stereotactic coordinates in the report (Meyer et al., 2003; Lindstrom et al., 2004; Ryding et al., 2006; Nye et al., 2013). One study was not included because the analyses focused only on striatal and cortical midline structures (Marchand et al., 2012), while another study was excluded as the study population consisted of elderly individuals (Dombrovski et al., 2013).

\section{ALE META-ANALYSIS}

Reported coordinates were analyzed for topographic convergence using the revised ALE algorithm for coordinate-based metaanalysis of neuroimaging results (Turkeltaub et al., 2002; Eickhoff et al., 2009). The goal of coordinate-based meta-analyses of neuroimaging data is to identify brain areas where the reported foci of activation converge across published experiments. To this end, the meta-analysis determines if the clustering is significantly higher than expected under the null distribution of a random spatial association of results from the considered experiments while acknowledging the spatial uncertainty associated with neuroimaging foci. As the first step, and following conversion from MNI space to Talairach space using icbm2tal where needed, reported foci were interpreted as centers for 3D Gaussian probability distributions that capture the spatial uncertainty associated with each focus. This uncertainty is mostly a function of between-template (attributable to different normalization strategies and templates across laboratories) and between-subject (due to small sample sizes) variance. In fact, the between-template and between-subject variability are acknowledged based on empirical estimates, the latter being additionally gaged by individual sample size (Eickhoff et al., 2009). In a second step, the probabilities of all activation foci in a certain experiment were combined for each voxel, yielding a modeled activation (MA) map (Turkeltaub et al., 2012). 
Voxel-wise ALE scores resulted from the union across these MA maps and quantified the convergence across experiments at each particular location in the brain. The third and last step distinguished between random and "true" convergence by comparing the ensuing ALE scores against an empirical null distribution reflecting a random spatial association between the experiments' MA maps (Eickhoff et al., 2012). The within-experiment distribution of foci, however, was regarded as fixed (Eickhoff et al., 2009). Thus, a random-effects inference was invoked, focusing on the above-chance convergence across different experiments (Eickhoff et al., 2009; Caspers et al., 2010; Kurth et al., 2010). The resulting ALE scores were tested against the earlier calculated "true" ALE scores and cut off at a cluster-level-corrected threshold of $p<0.05$. The cluster size threshold was $>200 \mathrm{~mm}^{3}$.

\section{RESULTS}

\section{STRUCTURAL IMAGING STUDIES}

As shown in Table 1, six structural imaging studies were included in the meta-analysis (Aguilar et al., 2008; Rusch et al., 2008; Jia et al., 2010; Benedetti et al., 2011; Mahon et al., 2012; Soloff et al., 2012).

The pooled study population of the six structural imaging studies in the meta-analysis consisted of 298 individuals, i.e., 116 suicide attempters, and 182 psychiatric controls. The total number of foci identified in the studies was 50. As shown in Figure 1, ALE analysis identified three clusters indicating reduced gray matter volume in the left hemisphere, i.e., in the caudate nucleus (cluster size: $360 \mathrm{~mm}^{3}$; center: $\left.x=-16,75, y=13,77, z=8,46\right)$, the superior temporal gyrus (cluster size: $280 \mathrm{~mm}^{3}$; center: $x=-41,49$, $y=-31,9, z=8,14$ ), and the rectal gyrus (cluster size: $240 \mathrm{~mm}^{3}$; center: $x=-9,93, y=35,81, z=-19,08)$.

\section{FUNCTIONAL IMAGING STUDIES}

As shown in Table 2, six studies were included in the meta-analysis (Jollant et al., 2008, 2010; Pan et al., 2011,2013a,b; Fan et al., 2013).

The pooled study population of the included functional imaging studies in the meta-analysis consisted of 177 individuals, i.e., 97 suicide attempters, and 80 psychiatric controls. The number of foci identified in the studies was 34. As shown in Figure 2, ALE analysis identified three clusters in the right hemisphere, i.e., two clusters in the right anterior cingulate and one in the posterior cingulate. Characteristics of the clusters in the right anterior cingulate were (1) cluster size: $480 \mathrm{~mm}^{3}$; center: $x=14,42, y=44,92$, $z=7,28$ (dorsal anterior cingulate), and (2) cluster size: $400 \mathrm{~mm}^{3}$; center: $x=13,73, y=11,28, z=43,55$ (rostral anterior cingulate). The size of the cluster in the right posterior cingulate was $384 \mathrm{~mm}^{3}$, centered at $x=2,97, y=-25,94, z=19,39$. The cluster in the dorsal anterior cingulate showed increased activation in suicide attempters when compared to psychiatric controls during exposure to angry faces or mild happy faces, while activation was relatively less increased in attempters than in psychiatric controls during high-risk decisions. The cluster in the rostral anterior cingulate showed increased activation in suicide attempters when compared to psychiatric controls during exposure to angry faces, while activation was relatively less increased in attempters than in psychiatric controls during a go/no-go task. Finally, the cluster in the posterior cingulate showed increased activation in suicide attempters when compared to psychiatric controls during exposure to happy faces, while activation was relatively less increased in attempters than in psychiatric controls during high-risk decisions.

\section{DISCUSSION}

This is the first meta-analysis of imaging studies of suicidal behavior. We were particularly interested in studying brain correlates of a vulnerability for suicidal behavior, and therefore included only studies, which reported a comparison between individuals with a history of a psychiatric disorder and a history of suicide attempts to individuals with a history of only this psychiatric disorder.

The findings support the existence of a vulnerability to suicidal behavior and suggest a neuroanatomical basis for this diathesis. In summary, ALE meta-analyses of 12 studies identifies six regions in the brain that in association with a history of suicidal behavior are characterized by decreased volumes or changes in reactivity to emotional and cognitive stimuli. The structural correlate of the vulnerability consisted of clusters of smaller volumes in the left superior temporal gyrus, rectal gyrus, and caudate nucleus. Functional correlates of the vulnerability to suicidal behavior were confined to the right cingulate gyrus, with two clusters in the anterior cingulate and one in the posterior cingulate.

Table 1 | Structural imaging studies meeting inclusion criteria.

\begin{tabular}{|c|c|c|c|c|c|c|c|}
\hline $\begin{array}{l}\text { Study } \\
\text { (first author) }\end{array}$ & Year & $\begin{array}{l}\text { Imaging } \\
\text { modality }\end{array}$ & $\begin{array}{l}\text { Study population } \\
\text { SA/PC/HC }\end{array}$ & $\begin{array}{l}\text { Mean age } \\
\text { years }\end{array}$ & $\% F$ & Measurement & Available data \\
\hline Aguilar & 2008 & MRI 1,5T & S $13 / 24 / 0$ & $37 / 43 / 0$ & $0 / 0 / 0$ & VBM & $\mathrm{BA} ; x, y, z ; \mathrm{T} ; p$ \\
\hline Rüsch & 2008 & $\mathrm{MRI} 1,5 \mathrm{~T}$ & S $10 / 45 / 55$ & $30 / 37 / 36$ & $30 / 40 / 38$ & VBM & $x, y, z ; p ;$ absolute volume (IU); $F ; p$ \\
\hline Jia & 2010 & MRI 3T & MD 16/36/52 & $34 / 35 / 37$ & $69 / 44 / 54$ & DTI FA & $x, y, z ;$ cluster size; $F ; t$ \\
\hline Benedetti & 2011 & MRI 3T & BD 19/38/0 & $44 / 46 / 0$ & $56 / 72 / 0$ & VBM & $\mathrm{BA} ; x, y, z ;$ cluster size; $z ; p$ \\
\hline Mahon & 2012 & $\mathrm{MRI} 1,5 \mathrm{~T}$ & BD 14/15/15 & $33 / 37 / 34$ & $36 / 40 / 47$ & DTI FA & $x, y, z ;$ cluster size; $p$ \\
\hline Soloff & 2012 & MRI 1,5T & BPD 44/24/0 & $30 / 26 / 26$ & $82 / 67 / 46$ & VBM & $x, y, z ;$ cluster size; $p$ \\
\hline
\end{tabular}

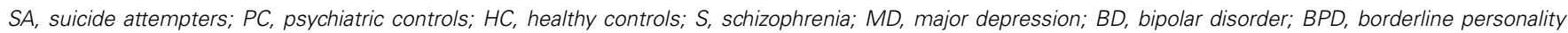

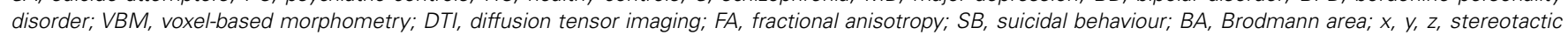
coordinates. 


-6 studies, 298 subjects (116 SA, $182 \mathrm{AC}$ ), 50 foci
-3 clusters identified
$\begin{aligned} & \text { Left caudate nucleus } \\ & (\mathrm{x}=-17, \mathrm{y}=14, \mathrm{z}=8) 360 \mathrm{~mm}^{3}\end{aligned}$
$\begin{aligned} & \text { BA } 41 \text { left superior temporal gyrus } \\ & (\mathrm{x}=-42, \mathrm{y}=-32, \mathrm{z}=8) 280 \mathrm{~mm}^{3}\end{aligned}$
B $\mathrm{x}=-10, \mathrm{y}=36, \mathrm{z}=-19) 240 \mathrm{~mm}^{3}$

Preceding a discussion of these findings a number of methodological issues need to be addressed. The number of studies, which could be included in the meta-analyses, is rather small. Due to methodological and ethical difficulties, inclusion of suitable suicidal patients in neurobiological research is a difficult process, so that it will take a substantial amount of time before a larger number of studies will be available for meta-analyses. The inclusion of a limited number of functional imaging studies implies that the effects of only a small number of activation paradigms have been investigated in suicide attempters. Therefore, it cannot be concluded that a diathesis to suicidal behavior is characterized by changed cingulate reactivity to particular facial emotional expressions or cognitive stimuli. Changes in such reactivity may also occur following exposure to other emotional or cognitive stimuli. In addition, it should be noted that studies were variable in terms of applied definitions of suicidal behavior and psychiatric diagnoses in suicide attempters and controls. The neurobiology of suicidal behavior along with depression may differ from that along with schizophrenia, but evidence of a shared underlying vulnerability is increasing (van Heeringen and Mann, 2014). As imaging studies involving elderly suicide attempters and controls were not included in the meta-analysis, the results of the current meta-analyses may not be applicable to the elderly. The conclusion that the demonstrated changes in brain functions and structures reflect a vulnerability to suicidal behavior is based on the assumption that suicide attempters and controls did not differ with regard to state-dependent characteristics. An effect of such state-dependent characteristics, including the use of medication, is most probably limited due to the strict inclusion criteria, but cannot be ruled out completely. Even with the strict inclusion criteria, the suitable studies still varied on a large number of variables such as statistical thresholds, smoothing kernels, registration procedures to standard space, and MRI scanning parameters. While current coordinate-based meta-analysis methods cannot account for all these differences separately, ALE does estimate a spatial uncertainty per individual study thereby alleviating some of the between-studies variability arising from varying study specific parameters such as the number of subjects or the use of different brain templates (Eickhoff et al., 2009). Finally, disturbances in brain functions may be attributable to structural changes. The differences between structurally and functionally affected regions in the current study, however, suggest that functional changes in association with a diathesis to suicidal behavior are not due to structural disturbances, at least not in the same areas. The relationship between the structural and functional findings will be discussed below.

With regard to the structural findings, combining voxel-based DTI and gray matter data in one meta-analysis may answer fundamental questions such as whether gray and white matter abnormalities, when found, are consistent with one another, and whether white matter alterations are consistent with alterations in gray matter of areas connected by these white matter abnormalities. The current meta-analysis, however, did not identify abnormalities in white matter in association with a history of suicidal behavior. A decreased gray matter volume of the orbitofrontal cortex has been shown in several psychiatric disorders, including depression (Wagner et al., 2008) and anxiety disorders (Strawn et al., 2013). Reduced volumes of the superior temporal gyrus have been demonstrated in schizophrenia (Palaniyappan et al., 2012). Reduced caudate volumes have been found in, among others, bulimia nervosa (Amianto et al., 2013), borderline personality disorder (O'Neill et al., 2013) and depression (Ma etal., 2012). These disorders also share a substantially increased suicide risk (Hawton and van Heeringen, 2009).

Table 2 | Functional imaging studies included in the meta-analysis.

\begin{tabular}{llllllll}
\hline $\begin{array}{l}\text { Study } \\
\text { (first author) }\end{array}$ & Year & $\begin{array}{l}\text { Imaging } \\
\text { modality }\end{array}$ & $\begin{array}{l}\text { Study population } \\
\text { SA/PC/HC }\end{array}$ & $\begin{array}{l}\text { Mean age } \\
\text { years }\end{array}$ & $\% \boldsymbol{F}$ & Measurement & Available data \\
\hline Jollant & 2008 & fMRI 1,5T & MD 13/14/16 & $40 / 44 / 32$ & $0 / 0 / 0$ & facial emotion recognition & BA; $x, y, z ;$ volume (voxels); $p$ \\
Jollant & 2010 & fMRI 1,5T & MD 13/12/- & $38 / 43 / 30$ & $0 / 0 / 0$ & IGT & BA; $x, y, z ; U ; p$ \\
Pan & 2011 & fMRI 3T & MD 15/15/14 & $16 / 16 / 15$ & $73 / 53 / 43$ & go/no-go & BA; $x, y, z ; F ; Z ; k$ (cluster); $p$ \\
Pan & $2013 a$ & fMRI 3T & MD 15/14/13 & $16 / 16 / 15$ & $73 / 50 / 38$ & IGT & BA; $x, y, z ; F ; Z ; k$ (cluster); $p$ \\
Fan & 2013 & fMRI 3T & MD 27/10/57 & $34 / 38 / 37$ & $59 / 56 / 55$ & ALFF & BA; $x, y, z ; K(c l u s t e r$ number); t \\
Pan & $2013 b$ & fMRI 3T & MD 14/15/15 & $16 / 16 / 15$ & $71 / 53 / 47$ & angry \& happy faces & BA; $x, y, z ; F ; Z ; k$ (cluster); t; $p$
\end{tabular}

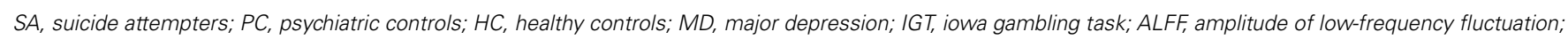
$B A$, Brodmann area; $x, y, z$, stereotactic coordinates. 


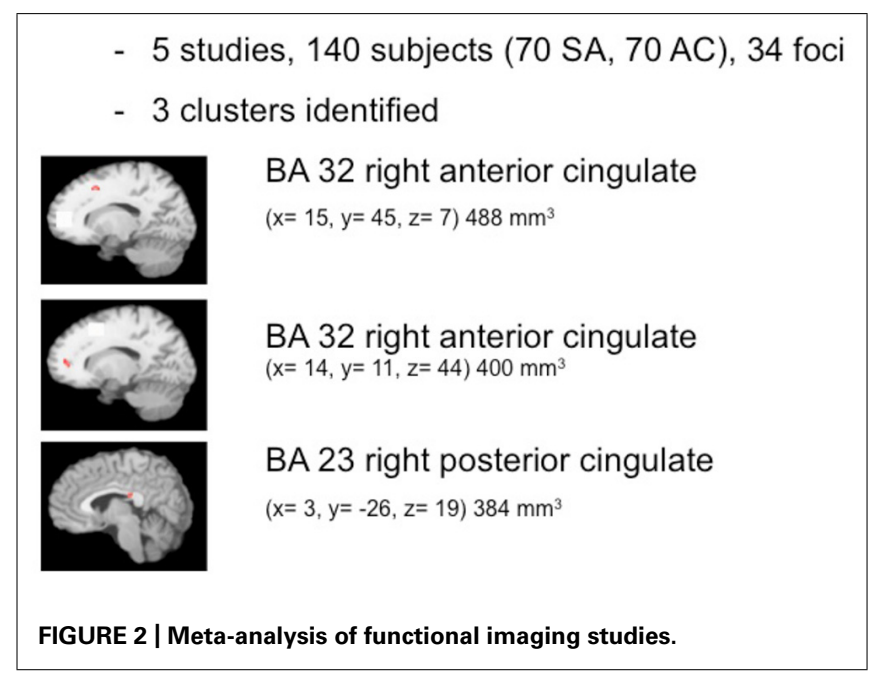

At first glance, the structural findings from the meta-analysis reflect a complex pattern of changes, and each of the involved regions may serve multiple functions. However, recent research findings point at a converging function from a cognitive neuroscience point of view, i.e., the processing of negative emotions. The superior temporal gyrus (Radua et al., 2010; Kumfor et al., 2013), the rectal gyrus (Schoenbaum et al., 2011; Szatkowska et al., 2011) and the caudate nucleus (Kemp et al., 2013) are involved in emotion processing, particularly with regard to negative emotions as shown in studies of facial emotion perception. The structures identified as structural correlates of the vulnerability to suicidal behavior appear to be particularly involved in the processing of the punishing aspect of salient events and may thus mediate in planning behavior on the basis of negative information.

The putative role of disturbed emotion processing, resulting in the aberrant salience of particular emotional stimuli, in the vulnerability to suicidal behavior is confirmed by the findings from the meta-analysis of functional imaging studies of suicidal behavior. Two clusters with changed activation patterns in association with a history of suicidal behavior were identified in the ACC, which is a structure in the medial prefrontal cortex that comprises several functional subdivisions. Rostral regions of the ACC (rACC) activate during emotional states or during tasks that involve interference from emotional stimuli. In contrast, dorsal regions of the ACC (dACC) activate during tasks that involve interference from non-emotional stimuli. The current meta-analysis shows increased activation during emotional tasks (i.e., exposure to emotional faces) and decreased activation during cognitive tasks [i.e., the iowa gambling task (IGT) and a Go/No-go task, respectively] in the rostral and in the dorsal ACC in association with a history of suicidal behavior. A distinction between rostral/emotional and dorsal/non-emotional is thus not found in this study, which is in keeping with recent models of functional organization of the brain (Lindquist and Barrett, 2012). A third cluster was identified in the PCC, in a similar way showing increased activation upon perception of emotional faces and decreased activation during the IGT.

The mechanism, by means of which reduced volumes of particular brain areas relate to changes in functional cognitive emotional characteristics, is yet unclear. Structural abnormalities may represent a trait factor and lead to functional changes that represent state factors (de Kwaasteniet et al., 2013). However, only very few studies have focused on the association between structural and functional cognitive or emotional alterations in the brain in the context of psychiatric disorders. No such studies exist with regard to suicidal behavior. Wagner et al. (2008) demonstrated a significant negative correlation between gray matter volume in the gyrus rectus and the BOLD signal in the rACC during the Stroop task in depressed individuals. Scheuerecker et al. (2010) reported a similar negative correlation between Brodmann area 11 (i.e., rectal gyrus) volumes and changes in the BOLD signal in, among others, the left caudate nucleus during an emotional face-matching task. The findings suggest that due to decreased gray matter in the orbitofrontal cortex depressed patients are not able to suppress interfering rACC and caudate activity. As the rACC and caudate are considered part of the brain's default mode network (DMN), the authors suggest that the inability to deactivate this network during cognitive processing is related to structural deficits in the rectal gyrus. Thus, a dysbalance of the orbitofrontal-cingulate network in controlling maladaptive affective responses during cognitive processing is strongly related to structural lesions within this network (Wagner et al., 2008). As the current meta-analysis showed similar functional disturbances in another part of the DMN, i.e., the PCC, the findings suggest that a vulnerability to suicidal behavior is associated with disturbances in an orbitofrontal- cingulate network, characterized by the interrelated inability to control maladaptive responses during cognitive processing and a reduced volume of the rectal gyrus. Grimm et al. (2009) provided further support for a role of reduced task-induced rACC DMN deactivation in suicidal behavior in depressed individuals by reporting a correlation between decreased negative BOLD responses in the DMN during emotion processing and feelings of hopelessness, a major risk factor for suicide. Thus, the current findings suggest that the increased salience of particular negative stimuli and the inability to control maladaptive responses during cognitive processing due to structural deficits are two core characteristics of the vulnerability to suicidal behavior.

Further insight in the nature of the deficits in cognitive processing associated with a vulnerability to suicidal behavior is provided by findings from neuropsychological studies. A recent meta-analysis showed a particular role of deficits in decisionmaking, verbal fluency and Stroop interference in this respect (Richard-Devantoy et al., 2014). While the currently demonstrated involvement of the ACC in a vulnerability to suicidal behavior may explain the lower Stroop performance, the findings from the current meta-analyses may particularly shed light on the role of deficient decision-making. The caudate, OFC and ACC are implicated in the process of reinforcement-guided decision-making, but appear to make distinctive contributions. In conjunction with the caudate, OFC codes the stimulus that is target of the action in terms of specific reward expectations, thus determining the tendency to action, i.e., to approach or avoid the predictive stimulus. Delay is an important aspect of relevant information for determining reward expectation. While coding of short-term reward expectation occurs in conjunction with the ventral striatum-based 
reward system, the dorsal striatum including the caudate nucleus appears to be involved in predicting future reward (Tanaka et al., 2004, 2007; Onoda et al., 2011). The dorsal striatum and its connected cortical control network thus enact motivational control over intentional behavior (Harsay et al., 2011). In keeping with this line of reasoning, a reduced caudate volume was recently found associated with increased delay discounting in Parkinson patients (Szamosi et al., 2013). Given the well-documented involvement of serotonin disturbances in suicidal behavior (Mann, 2013), it is of importance to note that striatal reward prediction at different time scales is modulated by the central serotonergic system (Tanaka et al., 2007).

Taken together, the findings from meta-analyses of neuroimaging and neuropsychological studies thus suggest that the vulnerability to suicidal behavior can be defined in terms of a reduced motivational control over the intentional behavioral reaction to salient negative stimuli. The current meta-analyses have identified structural abnormalities that may represent the vulnerability trait factor, leading to functional changes that may represent the state factors. Further study is needed to confirm these findings and explore network and connectivity characteristics of identified changes in neural substrates, the causes of these changes, which may be genetic or acquired, and the effects of treatments such as antidepressants, rTMS, ketamine, and psychotherapy on structural and functional changes.

\section{ACKNOWLEDGMENT}

The authors have neither financial interest in, nor financial support for writing this meta-analysis.

\section{REFERENCES}

Aguilar, E. J., Garcia-Marti, G., Marti-Bonmati, L., Lull, J. J., Moratal, D., Escarti, M. J., et al. (2008). Left orbitofrontal and superior temporal gyrus structural changes associated to suicidal behavior in patients with schizophrenia. Prog. Neuropsychopharmacol. Biol. Psychiatry 32, 1673-1676. doi: 10.1016/j.pnpbp.2008.06.016

Ahearn, E. P., Jamison, K. R., Steffens, D. C., Cassidy, F., Provenzale, J. M., Lehman, A., et al. (2001). MRI correlates of suicide attempt history in unipolar depression. Biol. Psychiatry 50, 266-270. doi: 10.1016/S0006-3223(01)01098-8

Amianto, F., Caroppo, P., D’Agata, F., Spalatro, A., Lavagnino, L., Caglio, M., et al. (2013). Brain volumetric abnormalities in patients with anorexia and bulimia nervosa: a Voxel-based morphometry study. Psychiatry Res. 213, 210-216. doi: 10.1016/j.pscychresns.2013.03.010

Audenaert, K., Goethals, I., Van Laere, K., Lahorte, P., Brans, B., Versijpt, J., et al. (2002). SPECT neuropsychological activation procedure with the Verbal Fluency Test in attempted suicide patients. Nucl. Med. Commun. 23, 907-916. doi: 10.1097/00006231-200209000-00015

Audenaert, K., Van Laere, K., Dumont, F., Slegers, G., Mertens, J., van Heeringen, C., et al. (2001). Decreased frontal serotonin 5-HT2a receptor binding index in deliberate self-harm patients. Eur. J. Nucl. Med. 28, 175182. doi: 10.1007/s002590000392

Benedetti, F., Radaelli, D., Poletti, S., Locatelli, C., Falini, A., Colombo, C., et al. (2011). Opposite effects of suicidality and lithium on gray matter volumes in bipolar depression. J. Affect. Disord. 135, 139-147. doi: 10.1016/j.jad.2011.07.006

Caspers, S., Zilles, K., Laird, A. R., and Eickhoff, S. B. (2010). ALE meta-analysis of action observation and imitation in the human brain. Neuroimage 50, 1148-1167. doi: 10.1016/j.neuroimage.2009.12.112

Cyprien, F., Courtet, P., Malafosse, A., Maller, J., Meslin, C., Bonafe, A., et al. (2011). Suicidal behavior is associated with reduced corpus callosum area. Biol. Psychiatry 70, 320-326. doi: 10.1016/j.biopsych.2011.02.035

de Kwaasteniet, B., Ruhe, E., Caan, M., Rive, M., Olabarriaga, S., Groefsema, M., et al. (2013). Relation between structural and functional connectivity in major depressive disorder. Biol. Psychiatry 74, 40-47. doi: 10.1016/j.biopsych.2012.12.024
Desmyter, S., van Heeringen, C., and Audenaert, K. (2011). Structural and functional neuroimaging studies of the suicidal brain. Prog. Neuropsychopharmacol. Biol. Psychiatry 35, 796-808. doi: 10.1016/j.pnpbp.2010.12.026

Dombrovski, A. Y., Siegle, G. J., Szanto, K., Clark, L., Reynolds, C. F., and Aizenstein, H. (2012). The temptation of suicide: striatal gray matter, discounting of delayed rewards, and suicide attempts in late-life depression. Psychol. Med. 42, 1203-1215. doi: 10.1017/S0033291711002133

Dombrovski, A. Y., Szanto, K., Clark, L., Reynolds, C. F., and Siegle, G. J. (2013). Reward signals, attempted suicide, and impulsivity in late-life depression. JAMA Psychiatry 70, 1020-1030. doi: 10.1001/jamapsychiatry.2013.75

Ehrlich, S., Breeze, J. L., Hesdorffer, D. C., Noam, G. G., Hong, X. N., Alban, R. L., et al. (2005). White matter hyperintensities and their association with suicidality in depressed young adults. J. Affect. Disord. 86, 281-287. doi: 10.1016/j.jad.2005.01.007

Ehrlich, S., Noam, G. G., Lyoo, I. K., Kwon, B. J., Clark, M. A., and Renshaw, P. F. (2004). White matter hyperintensities and their associations with suicidality in psychiatrically hospitalized children and adolescents. J. Am. Acad. Child Psychiatry 43, 770-776. doi: 10.1097/01.chi.0000120020.48166.93

Eickhoff, S. B., Bzdok, D., Laird, A. R., Kurth, F., and Fox, P. T. (2012). Activation likelihood estimation meta-analysis revisited. Neuroimage 59, 2349-2361. doi: 10.1016/j.neuroimage.2011.09.007

Eickhoff, S. B., Laird, A. R., Grefkes, C., Wang, L. E., Zilles, K., and Fox, P. T. (2009). Coordinate-based activation likelihood estimation meta-analysis of neuroimaging data: a random-effects approach based on empirical estimates of spatial uncertainty. Hum. Brain Mapp. 30, 2907-2926. doi: 10.1002/Hbm.20718

Fan, T. T., Wu, X., Yao, L., and Dong, J. (2013). Abnormal baseline brain activity in suicidal and non-suicidal patients with major depressive disorder. Neurosci. Lett. 534, 35-40. doi: 10.1016/j.neulet.2012.11.032

Giakoumatos, C. I., Tandon, N., Shah, J., Mathew, I. T., Brady, R. O., Clementz, B. A., et al. (2013). Are structural brain abnormalities associated with suicidal behavior in patients with psychotic disorders? J. Psychiatr. Res. 47, 1389-1395. doi: 10.1016/j.jpsychires.2013.06.011

Grimm, S., Boesiger, P., Beck, J., Schuepbach, D., Bermpohl, F., Walter, M., et al. (2009). Altered negative BOLD responses in the default-mode network during emotion processing in depressed subjects. Neuropsychopharmacol. 34, 932-943. doi: 10.1038/Npp.2008.81

Harsay, H. A., Cohen, M. X., Oosterhof, N. N., Forstmann, B. U., Mars, R. B., and Ridderinkhof, K. R. (2011). Functional connectivity of the striatum links motivation to action control in humans. J. Neurosci. 31, 10701-10711. doi: 10.1523/Jneurosci.5415-10.2011

Hawton, K., and van Heeringen, K. (2009). Suicide. Lancet 373, 1372-1381. doi: 10.1016/S0140-6736(09)60372-X

Hwang, J. P., Lee, T. W., Tsai, S. J., Chen, T. J., Yang, C. H., Lirng, J. F., et al. (2010). Cortical and subcortical abnormalities in late-onset depression with history of suicide attempts investigated with MRI and voxel-based morphometry. J. Geriatr. Psychiatry Neurol. 23, 171-184. doi: 10.1177/0891988710363713

Jia, Z. Y., Huang, X. Q., Wu, Q. Z., Zhang, T. J., Lui, S., Zhang, J. R., et al. (2010). High-field magnetic resonance imaging of suicidality in patients with major depressive disorder. Am. J. Psychiatry 167, 1381-1390. doi: 10.1176/appi.ajp.2010. 09101513

Jollant, F., Lawrence, N. L., Olie, E., Guillaume, S., and Courtet, P. (2011). The suicidal mind and brain: a review of neuropsychological and neuroimaging studies. World J. Biol. Psychia 12, 319-339. doi: 10.3109/15622975.2011.556200

Jollant, F., Lawrence, N. S., Giampietro, V., Brammer, M. J., Fullana, M. A., Drapier, D., et al. (2008). Orbitofrontal cortex response to angry faces in men with histories of suicide attempts. Am. J. Psychiatry 165, 740-748. doi: 10.1176/appi.ajp.2008.07081239

Jollant, F., Lawrence, N. S., Olie, E., O’Daly, O., Malafosse, A., Courtet, P., et al. (2010). Decreased activation of lateral orbitofrontal cortex during risky choices under uncertainty is associated with disadvantageous decision-making and suicidal behavior. Neuroimage 51, 1275-1281. doi: 10.1016/j.neuroimage.2010.03.027

Kemp, J., Berthel, M. C., Dufour, A., Despres, O., Henry, A., Namer, I. J., et al. (2013). Caudate nucleus and social cognition: neuropsychological and SPECT evidence from a patient with focal caudate lesion. Cortex 49, 559-571. doi: 10.1016/j.cortex.2012.01.004

Kumfor, F., Irish, M., Hodges, J. R., and Piguet, O. (2013). Discrete neural correlates for the recognition of negative emotions: insights from frontotemporal dementia. PLoS ONE 8:e67457 doi: 10.1371/journal.pone.0067457 
Kurth, F., Zilles, K., Fox, P. T., Laird, A. R., and Eickhoff, S. B. (2010). A link between the systems: functional differentiation and integration within the human insula revealed by meta-analysis. Brain Struct. Funct. 214, 519-534. doi: 10.1007/s00429010-0255-Z

Leyton, M., Paquette, V., Gravel, P., Rosa-Neto, P., Weston, F., Diksic, M., et al. (2006). Alpha-[C-11] Methyl-L-tryptophan trapping in the orbital and ventral medial prefrontal cortex of suicide attempters. Eur. Neuropsychopharmacol. 16, 220-223. doi: 10.1016/j.euroneuro.2005.09.006

Lindquist, K. A., and Barrett, L. F. (2012). A functional architecture of the human brain: emerging insights from the science of emotion. Trends Cogn. Sci. 16, 533-540. doi: 10.1016/j.tics.2012.09.005

Lindstrom, M. B., Ryding, E., Bosson, P., Ahnlide, J. A., Rosen, I., and TraskmanBendz, L. (2004). Impulsivity related to brain serotonin transporter binding capacity in suicide attempters. Eur. Neuropsychopharmacol. 14, 295-300. doi: 10.1016/S0924-977x(03)00218-9

Ma, C. Q., Ding, J. R., Li, J., Guo, W. B., Long, Z. L., Liu, F., et al. (2012) Resting-state functional connectivity bias of middle temporal gyrus and caudate with altered gray matter volume in major depression. PLoS ONE 7:e45263 doi: 10.1371/journal.pone.0045263

Mahon, K., Burdick, K. E., Wu, J. H., Ardekani, B. A., and Szeszko, P. R. (2012). Relationship between suicidality and impulsivity in bipolar I disorder: a diffusion tensor imaging study. Bipolar Disord. 14, 80-89. doi: 10.1111/j.1399-5618.2012.00984.x

Mann, J. J. (2013). The serotonergic system in mood disorders and suicidal behaviour. Philos. Trans. R Soc. B Lond. B Biol. Sci. 368, 20120537 doi: 10.1098/Rstb.2012.0537

Marchand, W. R., Lee, J. N., Johnson, S., Thatcher, J., Gale, P., Wood, N., et al. (2012). Striatal and cortical midline circuits in major depression: implications for suicide and symptom expression. Prog. Neuropsychopharmacol. Biol. Psychiatry 36, 290-299. doi: 10.1016/j.pnpbp.2011.10.016

Matsuo, K., Nielsen, N., Nicoletti, M. A., Hatch, J. P., Monkul, E. S., Watanabe, Y., etal. (2010). Anterior genu corpus callosum and impulsivity in suicidal patients with bipolar disorder. Neurosci. Lett. 469, 75-80. doi: 10.1016/j.neulet.2009.11.047

Meyer, J. H., McMain, S., Kennedy, S. H., Korman, L., Brown, G. M., DaSilva, J. N., et al. (2003). Dysfunctional attitudes and 5-HT2 receptors during depression and self-harm. Am. J. Psychiatry 160, 90-99. doi: 10.1176/appi.ajp.160.1.90

Monkul, E. S., Hatch, J. P., Nicoletti, M. A., Spence, S., Brambilla, P., Lacerda, A. L. T., et al. (2007). Fronto-limbic brain structures in suicidal and non-suicidal female patients with major depressive disorder. Mol. Psychiatry 12, 360-366. doi: 10.1038/sj.mp.4001919

Nery-Fernandes, F., Rocha, M. V., Jackowski, A., Ladeia, G., Guimaraes, J. L., Quarantini, L. C., et al. (2012). Reduced posterior corpus callosum area in suicidal and non-suicidal patients with bipolar disorder. J. Affect. Disord. 142, 150-155. doi: 10.1016/j.jad.2012.05.001

Nye, J. A., Purselle, D., Plisson, C., Voll, R. J., Stehouwer, J. S., Votaw, J. R., et al (2013). Decreased brainstem and putamen sert binding potential in depressed suicide attempters using [C-11]-zient pet imaging. Depress. Anxiety 30, 902-907. doi: 10.1002/Da.22049

O’Neill, A., D’Souza, A., Carballedo, A., Joseph, S., Kerskens, C., and Frodl, T. (2013). Magnetic resonance imaging in patients with borderline personality disorder: a study of volumetric abnormalities. Psychiatry Res. 213, 1-10. doi: 10.1016/j.pscychresns.2013.02.006

Onoda, K., Okamoto, Y., Kunisato, Y., Aoyama, S., Shishida, K., Okada, G., et al. (2011). Inter-individual discount factor differences in reward prediction are topographically associated with caudate activation. Exp. Brain Res. 212, 593-601. doi: 10.1007/s00221-011-2771-2773

Oquendo, M. A., Placidi, G. P. A., Malone, K. M., Campbell, C., Keilp, J., Brodsky, B., et al. (2003). Positron emission tomography of regional brain metabolic responses to a serotonergic challenge and lethality of suicide attempts in major depression. Arch. Gen. Psychiatry 60, 14-22. doi: 10.1001/archpsyc.60.1.14

Palaniyappan, L., Balain, V., Radua, J., and Liddle, P. F. (2012). Structural correlates of auditory hallucinations in schizophrenia: a meta-analysis. Schizophr. Res. 137, 169173. doi: 10.1016/j.schres.2012.01.038

Pan, L. A., Batezati-Alves, S. C., Almeida, J. R. C., Segreti, A., Akkal, D., Hassel, S., et al. (2011). Dissociable patterns of neural activity during response inhibition in depressed adolescents with and without suicidal behavior. J. Am. Acad. Child Adolesc. Psychiatry 50, 602-611. doi: 10.1016/j.jaac.2011.03.018
Pan, L. A., Hassel, S., Segreti, A. M., Nau, S. A., Brent, D. A., and Phillips, M. L. (2013a). Differential patterns of activity and functional connectivity in emotion processing neural circuitry to angry and happy faces in adolescents with and without suicide attempt. Psychol. Med. 43, 2129-2142. doi: 10.1017/S0033291712002966

Pan, L., Segreti, A., Almeida, J., Jollant, F., Lawrence, N., Brent, D., et al. (2013b). Preserved hippocampal function during learning in the context of risk in adolescent suicide attempt. Psychiatry Res. 211, 112-118. doi: 10.1016/j.pscychresns.2012.07.008

Pompili, M., Ehrlich, S., De Pisa, E., Mann, J. J., Innamorati, M., Cittadini, A., et al. (2007). White matter hyperintensities and their associations with suicidality in patients with major affective disorders. Eur. Arch. Psychiatry Clin. Neurosci. 257, 494-499. doi: 10.1007/s00406-007-0755-x

Pompili, M., Innamorati, M., Mann, J. J., Oquendo, M. A., Lester, D., Del Casale, A., et al. (2008). Periventricular white matter hyperintensities as predictors of suicide attempts in bipolar disorders and unipolar depression. Prog. Prog Neuropsychopharmacol. Biol. Psychiatry 32, 1501-1507. doi: 10.1016/j.pnpbp.2008.05.009

Radua, J., Phillips, M. L., Russell, T., Lawrence, N., Marshall, N., Kalidindi, S., et al. (2010). Neural response to specific components of fearful faces in healthy and schizophrenic adults. Neuroimage 49, 939-946. doi: 10.1016/j.neuroimage. 2009.08.030

Richard-Devantoy, S., Berlim, M. T., and Jollant, F. (2014). A meta-analysis of neuropsychological markers of vulnerability to suicidal behavior in mood disorders. Psychol. Med. 44, 1663-1673. doi: 10.1017/S0033291713002304

Rusch, N., Spoletini, I., Wilke, M., Martinotti, G., Bria, P., Trequattrini, A., et al. (2008). Inferior frontal white matter volume and suicidality in schizophrenia. Psychiatry Res. 164, 206-214. doi: 10.1016/j.pscychresns.2007.12.011

Ryding, E., Ahnlide, J. A., Lindstrom, M., Rosen, I., and Traskman-Bendz, L. (2006). Regional brain serotonin and dopamine transporter binding capacity in suicide attempters relate to impulsiveness and mental energy. Psychiatry Res. 148, 195203. doi: 10.1016/j.pscychresns.2006.06.001

Scheuerecker, J., Meisenzahl, E. M., Koutsouleris, N., Roesner, M., Schopf, V., Linn, J., et al. (2010). Orbitofrontal volume reductions during emotion recognition in patients with major depression. J. Psychiatry Neurosci. 35, 311-320. doi: 10.1503/Jpn.090076

Schoenbaum, G., Takahashi, Y., Liu, T. L., and McDannald, M. A. (2011). Does the orbitofrontal cortex signal value? Ann. N.Y. Acad. Sci. 1239, 87-99. doi: 10.1111/j.1749-6632.2011.06210.x

Soloff, P. H., Pruitt, P., Sharma, M., Radwan, J., White, R., and Diwadkar, V. A. (2012). Structural brain abnormalities and suicidal behavior in borderline personality disorder. J. Psychiatr. Res. 46, 516-525. doi: 10.1016/j.jpsychires.2012. 01.003

Spoletini, I., Piras, F., Fagioli, S., Rubino, I. A., Martinotti, G., Siracusano, A., et al. (2011). Suicidal attempts and increased right amygdala volume in schizophrenia. Schizophr. Res. 125, 30-40. doi: 10.1016/j.schres.2010.08.023

Strawn, J. R., Wehry, A. M., Chu, W. J., Adler, C. M., Eliassen, J. C., Cerullo, M. A., et al. (2013). Neuroanatomic abnormalities in adolescents with generalized anxiety disorder: a voxel-based morphometry study. Depress. Anxiety 30, 842848. doi: 10.1002/Da.22089

Szamosi, A., Nagy, H., and Keri, S. (2013). Delay discounting of reward and caudate nucleus volume in individuals with alpha-synuclein gene duplication before and after the development of Parkinson's disease. Neurodegener. Dis. 11, 72-78. doi: $10.1159 / 000341997$

Szatkowska, I., Szymanska, O., Marchewka, A., Soluch, P., and Rymarczyk, K. (2011). Dissociable contributions of the left and right posterior medial orbitofrontal cortex in motivational control of goal-directed behavior. Neurobiol. Learn. Mem. 96, 385-391. doi: 10.1016/j.nlm.2011.06.014

Tanaka, S. C., Doya, K., Okada, G., Ueda, K., Okamoto, Y., and Yamawaki, S. (2004). Prediction of immediate and future rewards differentially recruits cortico-basal ganglia loops. Nat. Neurosci. 7, 887-893. doi: 10.1038/ nn 1279

Tanaka, S. C., Schweighofer, N., Asahi, S., Shishida, K., Okamoto, Y., Yamawaki, S., et al. (2007). Serotonin differentially regulates short- and long-term prediction of rewards in the ventral and dorsal striatum. PLOS ONE 2:e1333 doi: 10.1371/Journal.pone.0001333

Turkeltaub, P. E., Eden, G. F., Jones, K. M., and Zeffiro, T. A. (2002). Meta-analysis of the functional neuroanatomy of single-word reading: method and validation. Neuroimage 16, 765-780. doi: 10.1006/nimg.2002.1131 
Turkeltaub, P. E., Eickhoff, S. B., Laird, A. R., Fox, M., Wiener, M., and Fox, P. (2012). Minimizing within-experiment and within-group effects in activation likelihood estimation meta-analyses. Hum. Brain Mapp. 33, 1-13. doi: 10.1002/Hbm.21186

Van Heeringen, C. (2012). "Stress-diathesis model of suicidal behavior," in The Neurobiological Basis of Suicide, ed. Y. Dwivedi (Boca Raton, FL: CRC Press), 113-125. doi: 10.1201/b12215-7

van Heeringen, C., Bijttebier, S., and Godfrin, K. (2011). Suicidal brains: a review of functional and structural brain studies in association with suicidal behaviour. Neurosci. Biobehav. R 35, 688-698. doi: 10.1016/j.neubiorev.2010.08.007

van Heeringen, K., and Mann, J. J. (2014). The neurobiology of suicide. Lancet Psychiatry 1, 63-72. doi: 10.1016/S2215-0366(14)70220-2

Vang, F. J., Ryding, E., Traskman-Bendz, L., van Westen, D., and Lindstrom, M. B. (2010). Size of basal ganglia in suicide attempters, and its association with temperament and serotonin transporter density. Psychiatry Res. Neuroimaging 183, 177-179. doi: 10.1016/j.pscychresns.2010.05.007

Wagner, G., Koch, K., Schactitzabel, C., Reichenbach, J. R., Sauer, H., and Schlosser, R. G. M. (2008). Enhanced rostral anterior cingulate cortex activation during cognitive control is related to orbitofrontal volume reduction in unipolar depression. J. Psychiatr. Neurosci. 33, 199-208.

Wagner, G., Koch, K., Schachtzabel, C., Schultz, C. C., Sauer, H., and Schlosser, R. G. (2011). Structural brain alterations in patients with major depressive disorder and high risk for suicide: evidence for a distinct neurobiological entity? Neuroimage 54, 1607-1614. doi: 10.1016/j.neuroimage.2010.08.082

Wagner, G., Schultz, C. C., Koch, K., Schachtzabel, C., Sauer, H., and Schlosser, R. G. (2012). Prefrontal cortical thickness in depressed patients with high-risk for suicidal behavior. J. Psychiatry Res. 46, 1449-1455. doi: 10.1016/j.jpsychires.2012.07.013
World Health Organisation [WHO]. (2002). World Report on Violence and Health, 1st Edn. Geneva: World Health Organisation.

Zhang, H., Chen, Z., Jia, Z., and Gong, Q. (2014). Dysfunction of neural circuitry in depressive patients with suicidal behaviors: a review of structrual and functional neuroimaging studies. Progr. Neuropsychopharmacol. Biol. Psychiatry 4, 61-66. doi: 10.1016/j.pnpbp.2014.03.002

Zuckerman, M. (1999). Vulnerability to psychopathology: A Biosocial Model. Washington, DC: American Psychological Association.

Conflict of Interest Statement: The authors declare that the research was conducted in the absence of any commercial or financial relationships that could be construed as a potential conflict of interest.

Received: 28 May 2014; accepted: 26 September 2014; published online: 22 October 2014.

Citation: van Heeringen K, Bijttebier S, Desmyter S, Vervaet M and Baeken C (2014) Is there a neuroanatomical basis of the vulnerability to suicidal behavior? A coordinatebased meta-analysis of structural and functional MRI studies. Front. Hum. Neurosci. 8:824. doi: 10.3389/fnhum.2014.00824

This article was submitted to the journal Frontiers in Human Neuroscience.

Copyright (c) 2014 van Heeringen, Bijttebier, Desmyter, Vervaet and Baeken. This is an open-access article distributed under the terms of the Creative Commons Attribution License (CC BY). The use, distribution or reproduction in other forums is permitted, provided the original author(s) or licensor are credited and that the original publication in this journal is cited, in accordance with accepted academic practice. No use, distribution or reproduction is permitted which does not comply with these terms. 